\title{
Simulation of delamination under impact using a global-local method in explicit dynamics
}

Article in Finite Elements in Analysis and Design · March 2017

DOI: $10.1016 /$ j.finel.2016.11.002

CITATIONS

0

5 authors, including:

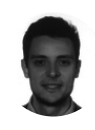

Omar Bettinotti

Dassault Systemes

9 PUBLICATIONS 11 CITATIONS

SEE PROFILE

Umberto Perego

Politecnico di Milano

159 PUBLICATIONS $\mathbf{1 , 1 1 3}$ CITATIONS

SEE PROFILE
READS

93
O. Allix

Ecole normale supérieure de Cachan

181 PUBLICATIONS 2,506 CITATIONS

SEE PROFILE

Benoît Malherbe

Airbus

15 PUBLICATIONS 21 CITATIONS

SEE PROFILE

Some of the authors of this publication are also working on these related projects: 


\title{
Simulation of delamination under impact using a global-local method in explicit dynamics
}

\author{
Omar Bettinotti $^{\mathrm{a}}$, Olivier Allix ${ }^{\mathrm{b}}$, Umberto Perego ${ }^{\mathrm{c}}$, Victor Oancea ${ }^{\mathrm{a}}$, Benoît \\ Malherbe $^{\mathrm{d}}$ \\ ${ }^{a}$ REDD Dassault Systèmes SIMULIA, 1301 Atwood Ave, 02919 Johnston RI, United \\ States of America. \\ ${ }^{b}$ LMT-Cachan, ENS-Paris-Saclay/CNRS/Pres UniverSud Paris, 61 av. du President \\ Wilson, 94235 Cachan, France. \\ ${ }^{c}$ Department of Civil and Environmental Engineering, Politecnico di Milano, p.zza \\ Leonardo da Vinci, 32, 20133 Milano, Italy. \\ ${ }^{d}$ Airbus, Vulnerability Tech Center, 316 Route de Bayonne, 31060 Toulouse, France.
}

\begin{abstract}
Despite of their interest, multi-scale methods based on domain decomposition are rarely used or even implemented within legacy codes. The reason is that their implementation is very demanding and that the robustness of their performance in industrial applications is questionable. In order to try to overcome these limitations, we recently adapted to the case of explicit dynamics a global-local multi-scale method (Bettinotti et al., 2014b). So far, the method has been implemented in a Matlab code and validated on simple elastic cases. In this paper, we present the implementation of the method in Abaqus/Explicit using its co-simulation features to couple two separate Abaqus/Explicit analyses, running at different scales. The approach is illustrated in the case of the simulation of delamination under high velocity impact. A key aspect of the method, if compared to the one based on domain decomposition, is the fact that the global model covers the whole structure. This feature has been used to treat contact at the global level only, which greatly simplifies the implementation and enhances the computational performance of the method. The effectiveness of the method has been verified by comparing the results with other approaches already available in Abaqus/Explicit: the tie constraint between different regions of the model and the sub-modeling approach.
\end{abstract}

Keywords: non-intrusivity, global-local approach, explicit dynamics, co-simulation, impact analysis, delamination. 


\section{Introduction}

The computationally effective simulation of large structures with complex non-linear local phenomena is still a scientific and industrial challenge. One of the main difficulties comes from the different length scales between the global response of the structure and the localized phenomena which can occur. To treat these questions in an effective manner, concurrent multi-scale methods have been developed first in statics. They are often based on domain decomposition techniques as the primal BDD method (Mandel, 1993), the dual FETI method (Farhat and Roux, 1991; Farhat et al., 1994), or the mixed Latin scheme (Ladevèze et al., 2001; Ladevèze and Nouy, 2003).

Due to the high cost of experiments, virtual testing is becoming essential for engineering workflows, as in the case of high velocity impact on composite structures, promoting the development of multi-scale methods also in dynamics. A number of studies have dealt with the extension of concurrent multi-scale methods to dynamics, leading to the design of multi-scale methods in space and time. Among them, some are based on non-uniform meshes and sub-cycling techniques, as in Belytschko et al. (1979) and Daniel (2003), others on the extension of the FETI decomposition method as in Gravouil and Combescure (2001) and following works. An extension of the mixed LATIN method in dynamics was also proposed in Boucard et al. (2002, 2011). These methods have proved all their potential in cases of local phenomena involving damage and failure, locally requiring short time increments and refined meshes, allowing to efficiently solve the induced vibration of the structure with much larger time increments and coarser meshes (Combescure and Gravouil, 2002).

Despite these needs, concurrent multi-scale methods are not widely employed in standard versions of legacy codes, even though different attempts have been made to introduce them. This is due both to the high cost associated to their implementation and to their lack of robustness with respect to the variety of situations, such as complex meshes, model coupling of different dimensions, type of elements and so on, which legacy codes have to deal with.

A possibility to overcome some of these limitations is to make use of localglobal approaches, where the global model extends over the whole structure

and is never changed, while the local model concerns only the small parts of the structure where non-local phenomena or high gradients are expected to 
take place. An advantage of such approaches is that a large part of the tools needed for their implementation is available in legacy codes. Local-global approaches basically perform iterative sub-modeling computations to converge toward the exact solution of the full global-local coupled model. A drawback of these methods is that they could require relaxation in order to converge. So far, these methods have mainly been developed in statics, following the earlier work of Whitcomb (1991). Non-intrusive substitution global-local coupling has been introduced in Gendre et al. (2009) and Gendre et al. (2011) for non-linear statics, in Plews et al. (2012) for thermo-mechanical coupling based on the Partition of Unity Method, or in Kerfriden et al. (2012), Gupta et al. (2012) and Passieux et al. (2013) for the simulation of fracture based on XFEM.

For dynamic applications, sub-modeling techniques (see Abaqus (2014)) are also available. They correspond to a sequentially-run global-to-local approach: the global analysis is run at first with a coarse mesh and associated large time increments, then the local analysis of a small part of the global structure, discretized with a fine mesh with its associated small time increments, is analyzed, driven by boundary conditions extracted and interpolated from the global solution. Such coupling can be defined "completely nonintrusive" because the implementation is simply based on an output-input data process between two analyses and "one-way coupling" because the data exchange is from global to local and the global solution is not corrected after the local one. Another available method in Abaqus is based on co-simulation. Co-simulation is generally employed for coupling two ore more software, for multi-scale and multi-physics problems.

A general co-simulation scheme is presented in Sicklinger et al. (2014) for coupling analyses with the same time increment. The domain decomposition method for implicit-explicit couplings through co-simulation is described in Chantrait et al. (2014), coupling Zebulon and Europlexus, or in Gigliotti and Pinho (2015), coupling Abaqus/Standard with Abaqus/Explicit. Also, an acoustic-dynamic coupling for automotive brake systems is described in Esgandari and Olatunbosun (2015), with Abaqus/Standard (i.e. the implicit version of the code) used for the complex eigenvalue analysis and Abaqus/Explicit for the finite elements dynamic analysis. A possible limitation of domain decomposition based multi-scale methods in dynamics is the requirement of a pre-subdivision and calibration of the model topology. In case of an evolutionary process, as in the case of delamination under impact, this would require the re-definition of the whole model, one of the most 
demanding tasks in engineering analysis of complex problems.

In order to try to circumvent this limitation, a concurrently-run, globallocal approach for explicit dynamics, which will be referred to in what follows as the "substitution method", has been introduced in Bettinotti et al. (2014a) as a weakly-intrusive method. In contrast to the sub-modeling technique, it is based on a two-way coupling exchange between the global coarse mesh, extending over the whole structure, and the fine mesh of a local small region, where a more detailed solution is required. Separate explicit dynamics analyses of the two meshes are concurrently run. The method allows each explicit analysis to march with its own time increment, dictated by the mesh size for stability requirements. As in sub-cycling techniques, the two analyses are synchronized at the end of the largest time increment ensuring that both interface velocities and accelerations are equal for each global time increment between the global and local scales. (see section 3.3.2). The equilibrium at the interface of the local domain is then achieved iteratively at each global time increment as explained in the paper. One appealing feature of explicit dynamics is that iterations concern the local domain only, the associated global modifications taking place at the next global time increment limiting therefore the cost of iterations, which, thanks to a reformulation in a more efficient iterative scheme in Bettinotti et al. (2014b), are limited between one and and three.

So far, the substitution method proposed in Bettinotti et al. (2014a) has been tested on simple examples using a Matlab prototype. In the case of linear elasticity, the comparison with the domain decomposition method, proposed in Gravouil and Combescure (2001) allowed to verify the method. In order to deal with problems of industrial interest, as the one of delamination under impact taken as reference example in this paper, the method has to be used within a legacy code, here the finite element code Abaqus/Explicit.

In statics, the Abaqus global and local models used in the proposed nonintrusive method can be coupled by making use of a Python script. This procedure would be much too costly in explicit dynamics where hundreds of thousands of time increments are often required even at the global level. The first problem to be considered is therefore the implementation of the method within the legacy code. The following questions need then be answered. What type of development does this require? How much of the standard capabilities available in Abaqus co-simulation engine can be taken advantage of? Does the proposed scheme offer a weakly intrusive way to be introduced in the core of a legacy code as it is expected? 
The second problem that we consider here concerns the difficulty exhibited by multi-scale methods in space and time based on domain decomposition in the case of constraints extending over different domains and integrated with different time increments. In that case, the finest time increment has to be used for every domain subject to the constraint, which limits the interest of those methods in quite a number of applications. For this reason, we investigate here the possibility to take a given constraint (here the contact condition) only at the global level. To examine these two issues, an example of interest for our partner Airbus has been chosen, the one of an impact of an object on a composite panel.

Besides the present introduction, the paper is organized according to the following scheme: section 2 will introduce the structural problem taken into exam and the model currently used by engineers, section 3 will present the multi-scale strategy with the introduction of the coupling to a coarser model to reduce computational cost and with the recall of the substitution method formulation, section 4 will show the results and validate the proposed approach with respect to other approaches already available in Abaqus/Explicit, like tie constraint and sub-modeling. Conclusions and prospects (section 5) close the paper.

\section{Structural test case}

\subsection{Modeling considerations for composite structures}

Let us consider a calibration test on a composite panel as the one described figure 1. If one wishes to predict directly the results of such an impact, we should ideally introduce in the modeling the different types of damage that happen at the so-called "meso-scale" of the ply (fiber failure, matrix cracking, fiber-matrix debonding) and at the interface between plies (see Allix (2001)).

What would the consequences of the use of such meso-scale model be? Let us consider a typical panel of $1 \mathrm{~m}^{2}$ with forty plies for a typical impact duration of three milliseconds. Each ply should be discretized separately, the typical scale being here a tenth of a millimeter, the interfaces between each plies should be discretized at least accordingly (and possibly with a finer mesh if one wish to properly describe the process zone at the delamination front). The associated finite element problem size corresponds to about three billions d.o.f.s, with some hundreds of thousands of time increments in ex- 
plicit dynamics. This is not the type of computation that can be performed today in engineering, that due to the lack of resources and time.

The procedure that is usually followed is based on the knowledge coming from previous tests on the same type of structures. The delaminated interfaces, usually one or two, are explicitly introduced in the model, while the other plies are stacked in multi-layered shell elements. We refer to this type of model as "pseudo-meso-scale" model. In this type of model for each stack, the plies stiffness is averaged through the thickness and governed by damage laws. Moreover, the interface parameters are calibrated in order to be able to use large elements depending on the number of plies. Such kind of ad-hoc model is capable to reduce by orders of magnitude the number of degrees of freedom when compared to meso-scale models. Typically the number of d.o.f. can be reduced to a hundred of thousand or so and the number of time increments to some ten of thousands. The limitation of such approaches is that they require an important a priori knowledge of the response of the structure.
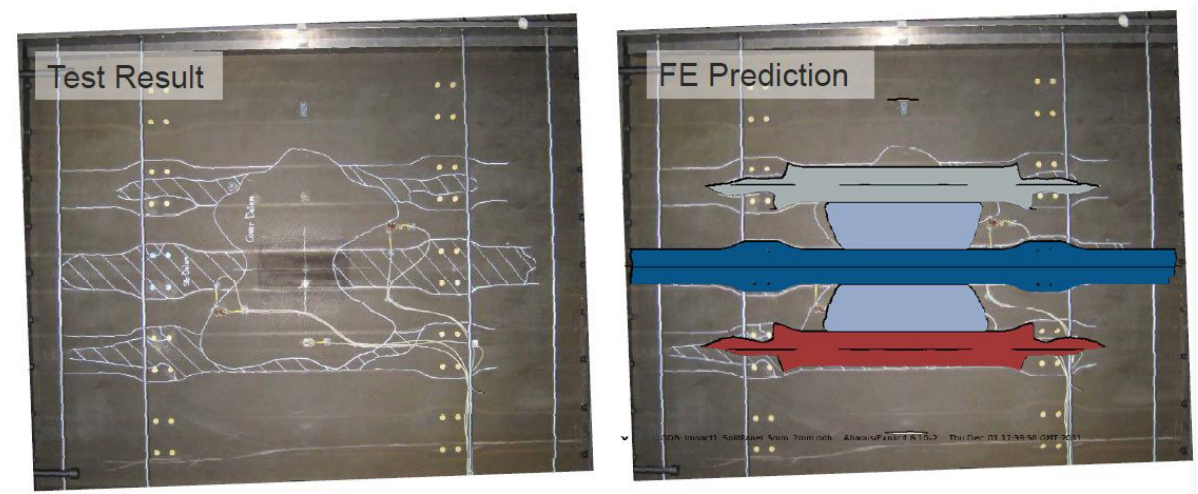

Figure 1: Vulnerability test case: reinforced composite panel impacted by tire debris rubber patch. Delamination is highlighted in both experimental and numerical analyses.

\subsection{Details of the considered test case}

The considered test case concerns a composite panel, which is unilaterally simply supported along the four edges. It is composed of 24 plies with the following fibers orientation sequence

$$
[45,-45,0,45,90,-45,0,0,45,0,0,-45 \mid \ldots \text { symm. }]
$$


for a total thickness of $6 \mathrm{~mm}$. The in-plane panel sizes are $0.45 \mathrm{~m}$ along the direction of the vertical edges and $0.5 \mathrm{~m}$ along the other direction. The rubber patch has size $0.088 m \times 0.102 m$ in its impacting surface and its projected edges on the composite plate are rotated by $-11^{\circ}$ with respect to the plate. The rubber patch initially lays in a plane not parallel to the plane of the panel, and the impact occurs at first along the left edge of the rubber patch. The initial inclination is $3^{\circ}$ with respect to the panel plane. The coarsest discretization model capable to predict delamination is the pseudo-meso-scale model shown in Figure 2. It consists of two layers of continuum shell elements through the panel thickness connected by a cohesive interface in correspondence of the panel middle plane. The in-plane size of the continuum shell elements is $5 \times 5 \mathrm{~mm}^{2}$, whereas the in-plane size of the cohesive elements is $2 \times 2 \mathrm{~mm}^{2}$. Due to the meshes mismatch, a surface-based tie constraint (see chapter 35.3.1 of Abaqus (2014) for details) is employed to connect the surfaces without coincident nodes. The tie constraint technique is used to connect together surfaces with non-coincident nodes: slave and master surfaces need to be defined a priori and slave nodes are subjected to forces obtained from the master nodes, such that the given slave nodes are constrained onto given positions over the master surface. Slave nodes degrees of freedom are then expressed in terms of master nodes ones and eliminated.

The different size of shell and interface cohesive elements is motivated by the need to limit the computational cost by reducing the number of degrees of freedom and, at the same time, by the need to resolve the length of the delamination process zone with the interface cohesive elements. This is considered an acceptable compromise between efficiency and accuracy.

The composite structure is modeled through the thickness by two damageable layers, each one made of 12 plies. Each layer obeys the intra-ply damage initiation criterion of Hashin and Rotem (1973). A cohesive interface is interposed between the two layers. Such cohesive interface obeys the damage initiation criterion based on the quadratic nominal stress defined as

$$
\left(\frac{\sigma_{n}}{\sigma_{n}^{0}}\right)^{2}+\left(\frac{\sigma_{t}}{\sigma_{t}^{0}}\right)^{2}+\left(\frac{\sigma_{s}}{\sigma_{s}^{0}}\right)^{2}=1
$$

where $\sigma$ denotes nominal stress, subscripts $n, t$ and $s$ denote normal and tangent directions, respectively, superscript 0 denotes maximum permissible values. 


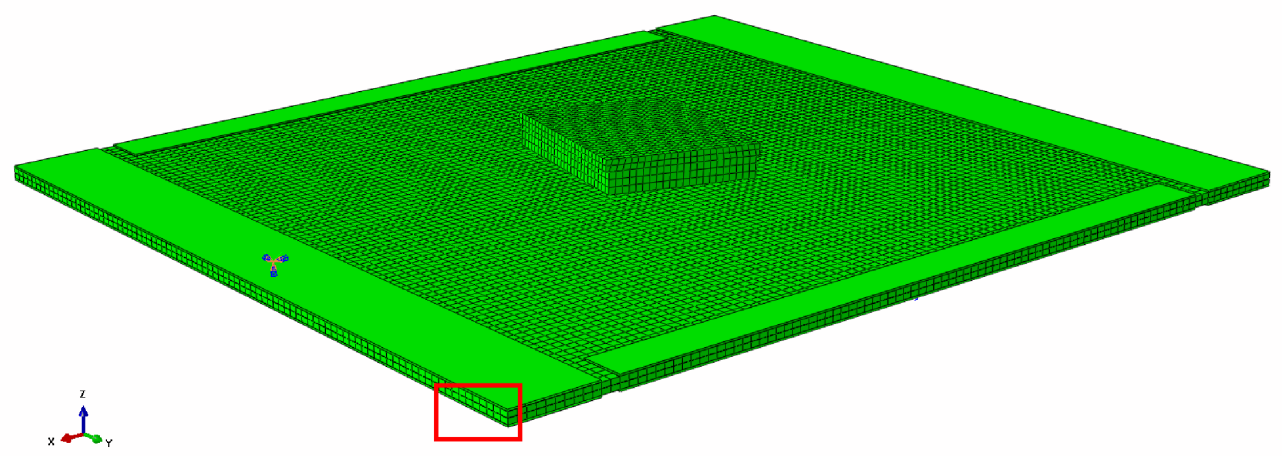

(a) mesh of the whole panel

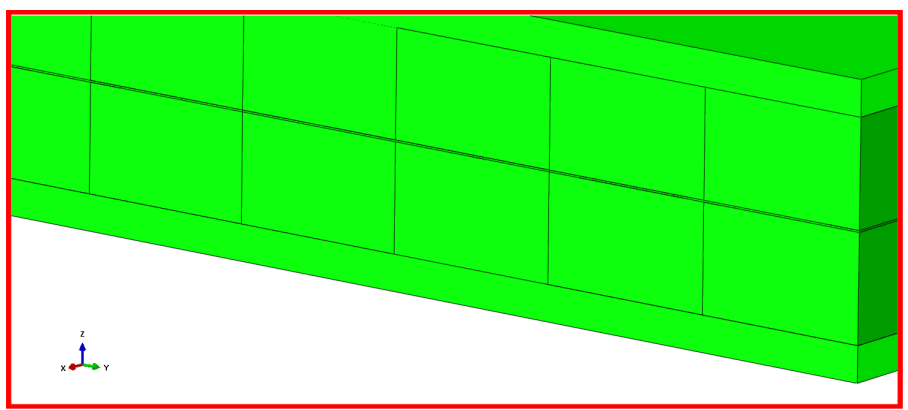

(b) enlarged view of panel corner in correspondence of supports

\begin{tabular}{|c|c|c|}
\hline No. & Orientation & Thickness \\
\hline Ply-24 & 45 & 0.25 \\
\hline Ply-23 & -45 & 0.25 \\
\hline Ply-22 & 0 & 0.25 \\
\hline Ply-21 & 45 & 0.25 \\
\hline Ply-20 & 90 & 0.25 \\
\hline Ply-19 & -45 & 0.25 \\
\hline Ply-18 & 0 & 0.25 \\
\hline Ply-17 & 0 & 0.25 \\
\hline Ply-16 & 45 & 0.25 \\
\hline Ply-15 & 0 & 0.25 \\
\hline Ply-14 & 0 & 0.25 \\
\hline Ply-13 & -45 & 0.25 \\
\hline \multicolumn{3}{|c|}{ Cohesive layer } \\
\hline Ply-12 & -45 & 0.25 \\
\hline Ply-11 & 0 & 0.25 \\
\hline Ply-10 & 0 & 0.25 \\
\hline Ply-9 & 45 & 0.25 \\
\hline Ply-8 & 0 & 0.25 \\
\hline Ply-7 & 0 & 0.25 \\
\hline Ply-6 & -45 & 0.25 \\
\hline Ply-5 & 90 & 0.25 \\
\hline Ply-4 & 45 & 0.25 \\
\hline Ply-3 & 0 & 0.25 \\
\hline Ply-2 & -45 & 0.25 \\
\hline Ply-1 & 45 & 0.25 \\
\hline & \multicolumn{2}{|c}{} \\
\hline
\end{tabular}

(c) composite plies

Figure 2: Pseudo-meso-scale model. a) Plies thickness and orientation. b) Detail of discretized panel thickness. c) Overview of the discretized model, including the rubber patch. 
Figure 3 shows the model considered for the numerical simulation of the impact test case. Four rigid bars along the edges support the panel. The position of the bars is shown in Figure 3. The sizes of the horizontal bars are $0.025 \mathrm{~m}$ of width and $0.38 \mathrm{~m}$ of length. The sizes of the vertical bars are $0.05 \mathrm{~m}$ of width and $0.45 \mathrm{~m}$ of length. Figure $3 \mathrm{~b}$ shows a detail of the supported edge. The simple support is achieved by inserting the plate side into the groove defined by the rigid support system. Contact with friction between the plate and the rigid supports is defined (with a friction coefficient of 0.4). The tire debris rubber patch is modeled as an Ogden hyper-elastic

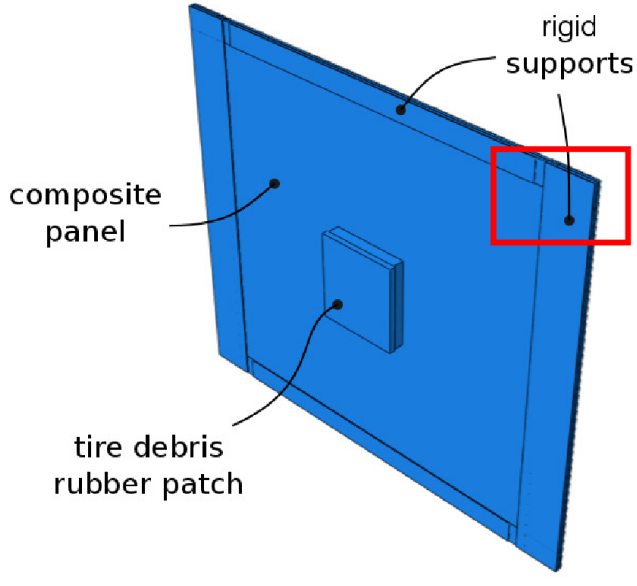

(a) overall panel and impactor

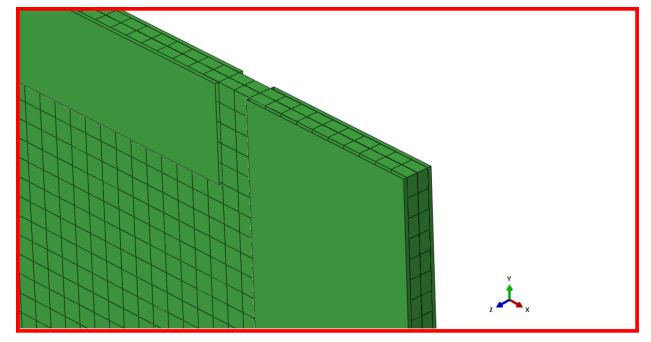

(b) detail of the rigid bars

Figure 3: Impact test case. a) Stratified composite panel impacted by tire debris rubber patch. b) Details of mesh and constraints.

material using the model available in Abaqus. A contact model is also defined for the interactions between panel and tire debris (with a friction coefficient of 0.3$)$.

The use of this coarse finite element model, with a total of 77246 elements, 154170 nodes, 462516 variables for an impact history of $0.2 \mathrm{~ms}$ requires a CPU-time of 7 min $45 s$ (1-CPU) and a memory of $518.2 \mathrm{Mb}$ on a Intel(R) Core(TM) i7-4800MQ CPU@2.7GHz with 16Gb RAM. For the purposes of this paper, i.e. for the comparison of existing multi-scale methodologies in simulating delamination the analysis is conducted during $0.2 \mathrm{~ms}$ which is sufficient to obtain a significant delamination. 


\section{Multi-scale coupling}

A multi-scale coupling is then considered in order to reduce the computing costs. In this case, the pseudo-meso-scale model described above is limited to a restricted region that includes the impacted area, assuming that the delamination process zone is activated and propagates in a limited zone before $t=0.2 \mathrm{~ms}$. A coarser "macro-scale" model is applied to the rest of the structure, as shown in Figure 4. The impact happens first in the central zone of the plate, but the rubber patch is inclined with respect to the plate middle plane. For this reason, the pseudo-meso-scale model is applied to an area of size $220 \times 220 \mathrm{~mm}^{2}$ but off-center of $20 \mathrm{~mm}$ along the $X$ direction and $15 \mathrm{~mm}$ along the $Y$ direction (see Figure 4 for $X-Y$ directions definition).

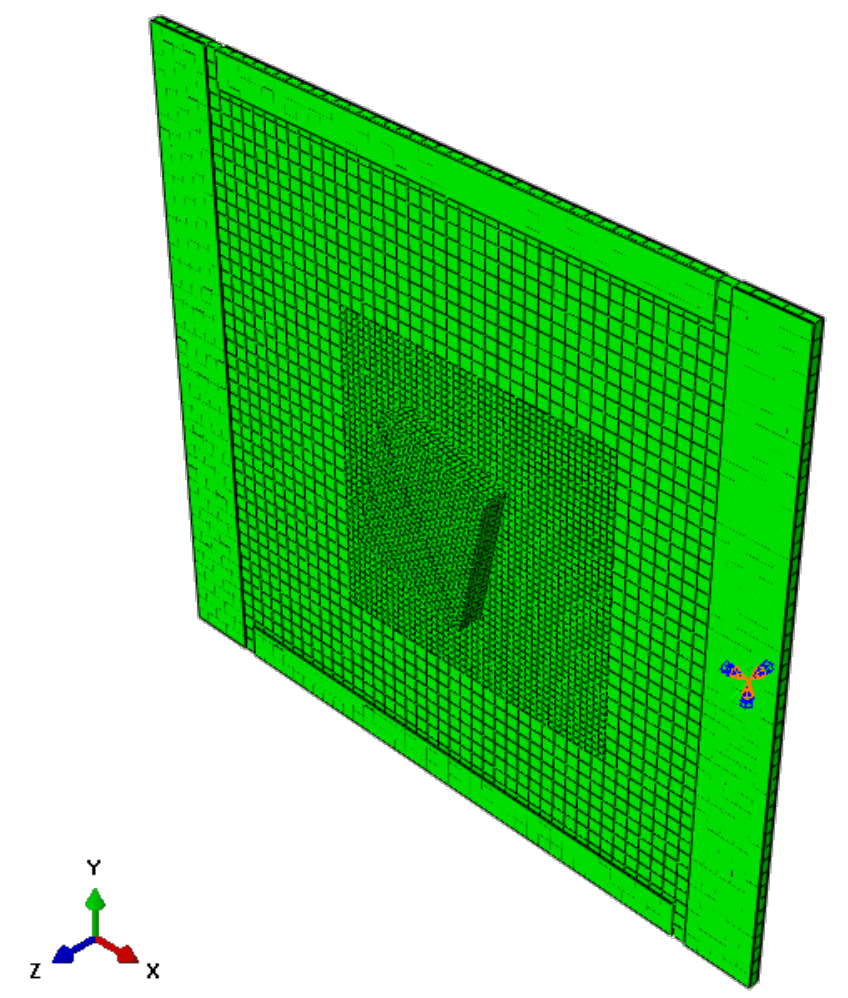

Figure 4: Non-uniform mesh with macro- and pseudo-meso-scale models.

The macro-scale model is made of just one continuum shell element through the thickness of the panel (see Figure 5) and is also assumed to 
be linear elastic, with a substantial reduction of computational costs. The non-linear behavior of the structure is considered only in the panel region discretized using the pseudo-meso-scale model. Exploiting the linear elastic behavior, the homogeneized shell section stiffness properties, equivalent to the layered composite, can be pre-computed only once by averaging through the thickness the plies properties (see e.g. chapter 29.6.6 of Abaqus (2014) for details).

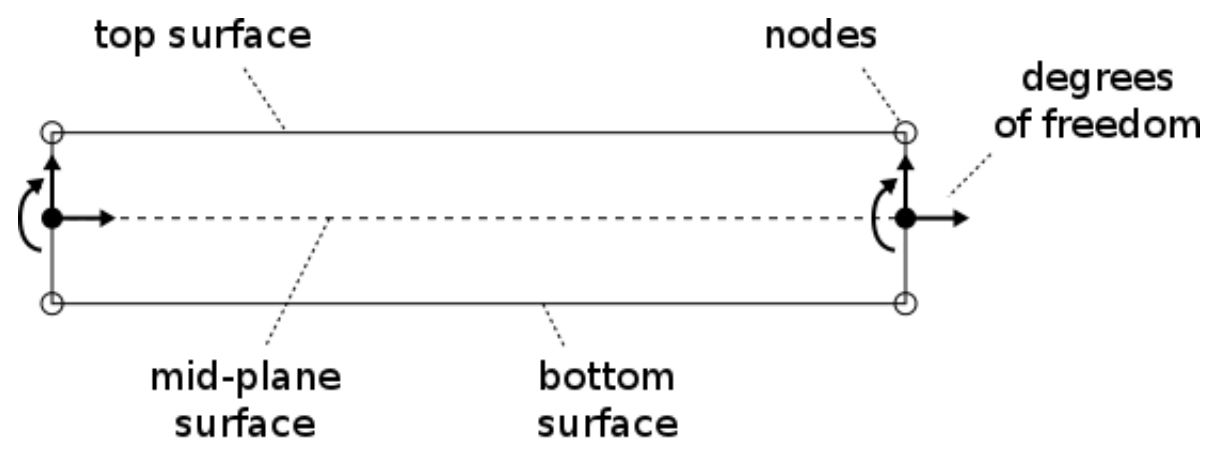

Figure 5: Continuum shell section: the mid-plane degrees of freedom are mapped onto the top-bottom nodes.

The in-plane size of the continuum shells in the macro-scale problem are chosen as $10 \times 10 \mathrm{~mm}^{2}$, in order to resolve with acceptable approximation the length of the stress waves generated during the impact.

\subsection{Application of the tie constraint technique}

The mesh mismatch between the macro- and pseudo-meso-scale models (the two models have conforming shapes but non-conforming meshes) requires a special treatment to guarantee compatibility. A first possibility consists of the application of a surface-based tie constraint (see chapter 35.3.1 of Abaqus (2014) for details and section 2 for a first description and application). As mentioned before, tie constraint allows non-coinciding nodes onto a common surface to be tied together for the duration of the analysis. In this particular case, depicted in Figure 4, the macro- and pseudo-mesoscale models have four through-the-thickness surfaces in common, in which coinciding nodes reside on the the plate top and bottom surfaces and noncoinciding nodes over the plate mid-plane, which belongs to the pseudo-mesoscale model. Figure 6 shows a detail of the tie constraint scheme, highlighting master and slave nodes. A non-coinciding node between macro- and pseudo- 


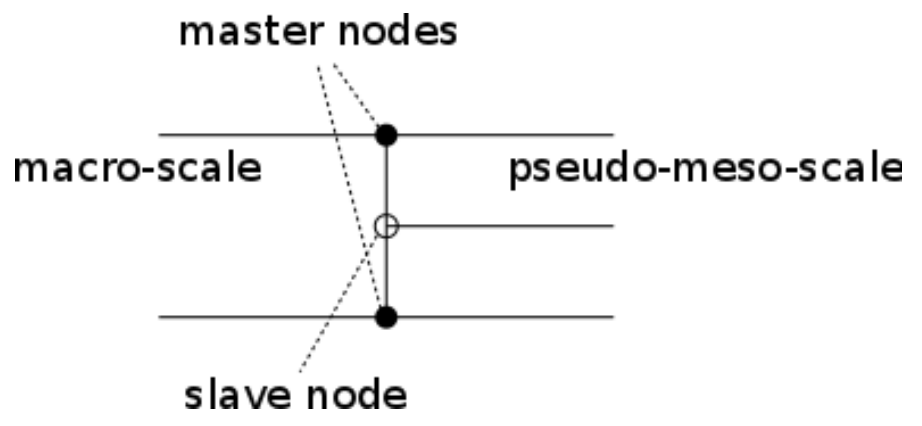

Figure 6: Tie constraint scheme between macro- and pseudo-meso-scale meshes.

meso-scale meshes, denoted as slave node in Figure 6, is constrained to the two coinciding nodes, the so-called master nodes. The slave node degrees of freedom are corrected at the end of the time increment. A unique time increment size is uniformly employed in the overall structure, due to a limitation of the tie constraint technique. Such size is determined at every time increment based on the smallest element size, so that the pseudo-meso-scale model dictates the time incrementation.

\subsection{Application of the sub-modeling technique}

The sub-modeling technique (see chapter 10.2.1 of Abaqus (2014) for details) is a global-local approach based on a sequence of analyses from the coarsest to the most refined one.

In this particular test case, a global analysis is run first with the macroscale model of the overall panel, as depicted in Figure 7a. Then, the related solution history in terms of displacements is extracted and interpolated over the global-local interface and applied as boundary condition in the subsequent local analysis, which is shown in Figure 7, with the pseudo-meso-scale model.

Each analysis employs a different mesh, whose average size is dictated by the problem size and by the available computing resources. Except for the boundary conditions in the local analysis, the two analyses are independent of each other and have two different time incrementations calculated on the basis of the element sizes. Furthermore, the definition of contact and of the tire rubber patch impactor have to be defined in both analyses, because there is no possibility to extract and interpolate the loading information from global to local. 


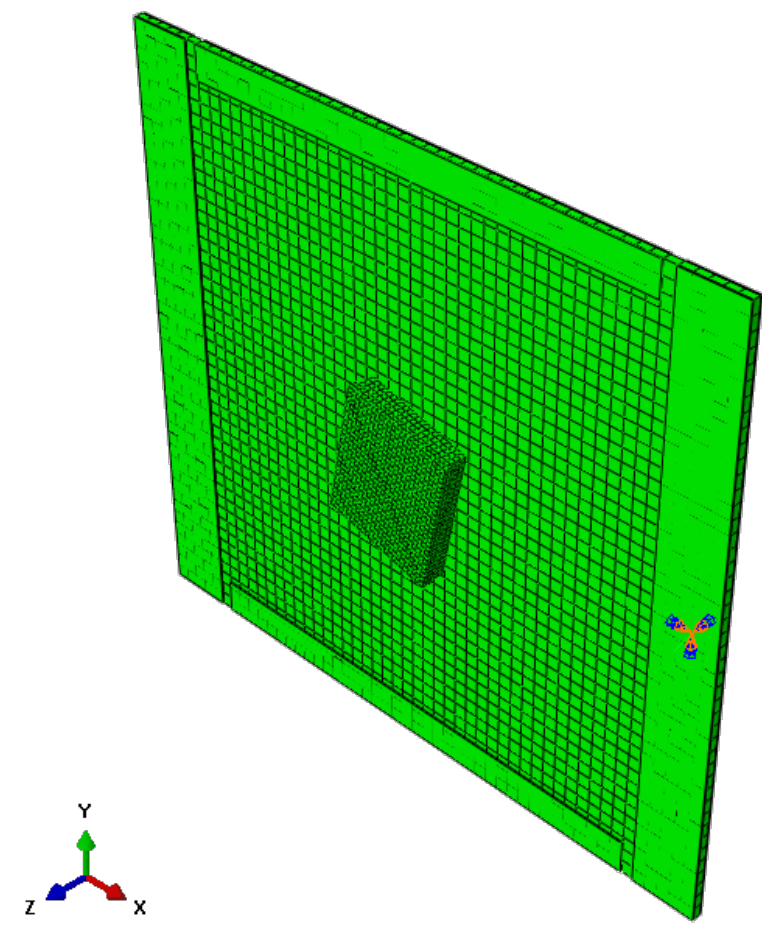

(a) global mesh applied to the whole panel

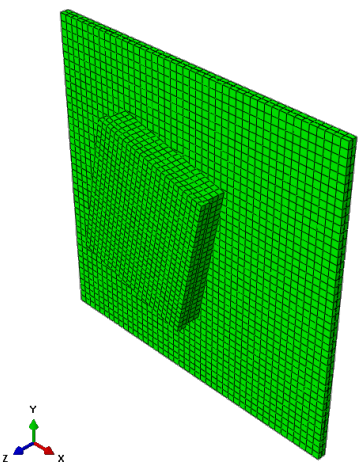

(b) local patch

Figure 7: Sub-modeling technique: sequentially-run global and local analyses.

As mentioned before, the sub-modeling technique is considered as nonintrusive because the coupling is extremely simple and based on the transmission of boundary results from the global to the local analysis. On the other hand, the local solution is accurate only if the local response does not have an appreciable influence on the global analysis, because the local analysis is driven by the global solution, but the global solution is not corrected by the local analysis.

\subsection{Implementation and application of the "substitution method"}

In contrast to sub-modeling, the substitution method is a global-local approach in which the two analyses are run concurrently and iteratively, exchanging information until convergence is reached. The main idea in the substitution method is to run an analysis of the whole structure under applied loads using a coarse mesh, compatible with the available computing resources. 
Then, a refined mesh of a local region of limited size, where more detailed information or a more accurate solution is required, is constructed. The part of the boundary of the local region which cuts the global mesh in two partitions is called "interface". An analysis of the local region is carried out with boundary conditions on the interface extracted from the solution of the global analysis. The solution on the boundary of the local region is then used to improve the accelerations at the nodes of the global mesh belonging to the interface. The global analysis is run again with new equivalent nodal forces applied along the interface. The process is repeated iteratively until convergence is reached. The data exchange through the interface of the global and local domains is carried out at each global time increment using the cosimulation technique (see chapter 27.1.1 of Abaqus (2014)), that allows one to couple different software together. The substitution method is so depicted as a "two-way coupling" sub-modeling technique, because the communication occurs in both ways, from global to local and viceversa.

The global analysis of the impact problem described in the previous section is carried out by modeling the entire plate domain $\Omega_{g}^{h}$ with the macroscale mesh and the time increment size is computed based on the macro-scale element size. The pseudo-meso-scale model concerns the local region $\Omega_{\ell}^{h}$ and it requires a smaller time increment, so that the coupling is also based on a sub-cycling scheme. As shown in Figure 8, the global analysis can use a large time increment $\Delta t_{g}=t^{n+1}-t^{n}$ while the local analysis is sub-cycling with smaller time increments $\Delta t_{\ell}=t^{m+1}-t^{m}$. The few last local time increments of the local analysis are adjusted so as to match the final instant of the global time increment $t^{n+1}$.

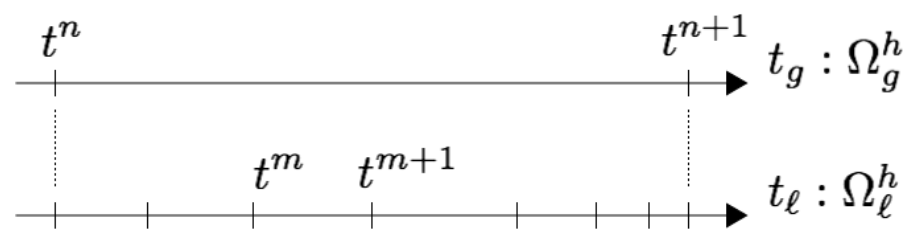

Figure 8: Time sub-cycling with arbitrary time increments.

Figure 9 illustrates in a schematic way, with reference to a hypothetical 2D problem, the definitions of the various geometrical entities required in the global-local substitution scheme. The global model mesh is coarser than the local one, where a refinement is in general necessary due to either fine geometrical details or nonlinear structural behavior inducing high stress/strain 
gradients. As in the test case considered in Figure 1, the structure can be subjected to contact with other objects (orange in the figure). The search for contact and the definition of the contact forces can in principle be conducted either at the global or local scale. In the proposed scheme, the contact search is carried out at the global level and the contact forces are applied to the coarse mesh in the global analysis. Also other possible forces applied on the structural boundary are considered at the global level. In summary, while contact and other external boundary forces are considered in the global analysis, material nonlinearities and refined geometrical features are considered in the local model.

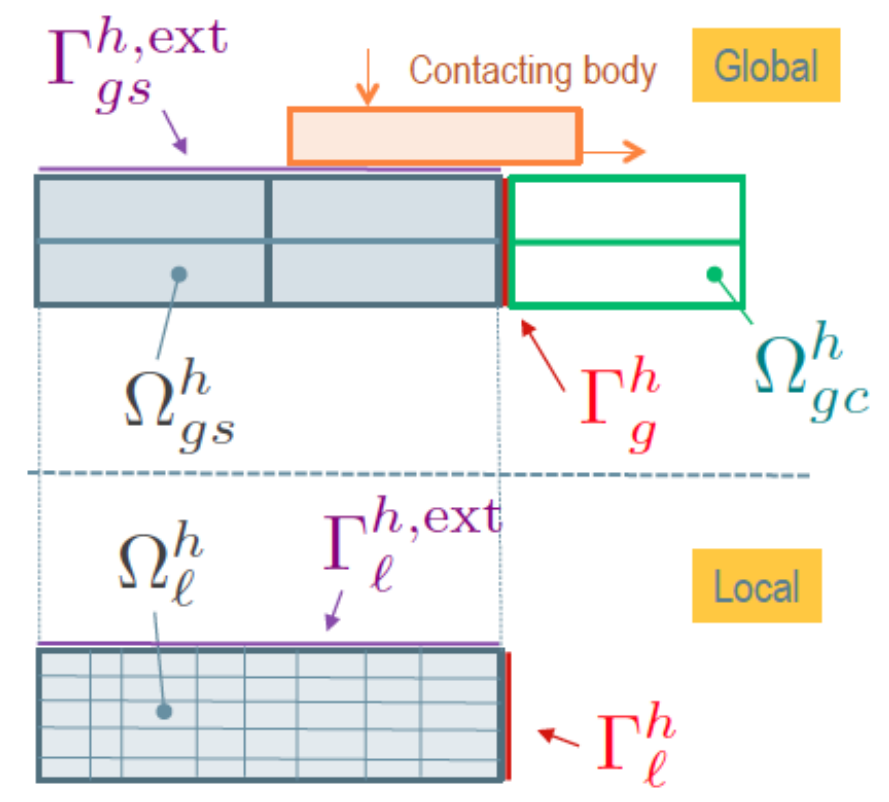

Figure 9: Multi-scale scheme for substitution via co-simulation technique.

Figure 9 is divided into two parts by a horizontal dashed line. The upper part, shows the coarse global model, where contact can occur between separate bodies. The global model, denoted by subscript $g$, is partitioned into two regions: the global substitution region $\Omega_{g s}^{h}$ (light gray in the figure) and the global complementary region $\Omega_{g c}^{h}$ white with green boundary in the figure), which are connected by the global interface $\Gamma_{g}^{h} \equiv \partial \Omega_{g s}^{h} \cap \partial \Omega_{g c}^{h}$. In a general situation, the contact and other loaded parts of the boundary surface may extend over parts of both $\partial \Omega_{g s}^{h}$ and $\partial \Omega_{g c}^{h}$. The parts of the boundary of 
the substitution region where contact forces and other boundary forces are applied is denoted as $\Gamma_{g s}^{h \text {,ext }} \subset \partial \Omega_{g s}^{h}$ (purple in the figure). Equivalent nodal contact forces applied to this surface will be mapped onto the corresponding surface $\Gamma_{\ell}^{h, \text { ext }}$ of the local mesh. The lower part of the figure, below the dashed line, shows the model of the local domain $\Omega_{\ell}^{h}$. It consists of a refined discretization of the domain $\Omega_{g s}^{h}$ of the global model, shown in the upper part of the figure. $\Omega_{g s}^{h}$ is called "substitution region" because the global solution in that region will be replaced by the more accurate one obtained by solving the refined problem on $\Omega_{\ell}^{h}$. The local interface $\Gamma_{\ell}^{h}$ (red in the figure) coincides with the global one $\Gamma_{g}^{h}$, as well as the loaded surface $\Gamma_{\ell}^{h, \text { ext }}$ coincides with $\Gamma_{g s}^{h, \text { ext }}$.

Contact forces are mapped in space and time, through linear interpolation, from the global surface $\Gamma_{g s}^{h, \text { ext }}$ to the local one $\Gamma_{\ell}^{h \text {,ext }}$ (purple surfaces in Figure 9). As a consequence, contact is modeled in the local analysis as application of external forces, varying in time according to the global solution. Details will be given in section 3.3.4.

With the definitions of Figure 9, the co-simulation technique implements the following data exchange at each global time increment:

- for the iterative coupling through surfaces $\Gamma_{g}^{h}$ and $\Gamma_{\ell}^{h}$ (based on the algorithm presented in Bettinotti et al. (2014b), whose details will be recalled in 3.3.2):

- the global analysis sends and maps to the lower scale velocities and accelerations to be applied to the local analysis as boundary conditions;

- the local analysis sends and maps to the higher scale mass and equivalent external and internal nodal forces acting on the interface $\Gamma_{\ell}^{h}$, in order to correct the solution in the global analysis;

- for the direct substitution of the solution in domain $\Omega_{g s}^{h}$ by the solution in $\Omega_{\ell}^{h}$ (whose details will be given in 3.3.3):

- the local analysis sends and maps velocities to be applied at nodes of the substitution region at the global scale.

- for the exchange of the external loads and contact information through surfaces $\Gamma_{g s}^{h, \text { ext }}$ and $\Gamma_{\ell}^{h \text {,ext }}$ (whose details will be given in 3.3.4): 
- the global analysis sends and maps equivalent nodal contact and external forces to be applied to the local analysis;

\subsubsection{Code implementations in Abaqus/Explicit}

New code implementations, not available in the standard commercial version, have been necessary in Abaqus/Explicit in order to integrate the algorithm into the co-simulation services and into the Abaqus/Explicit application.

Four services have been implemented in Abaqus/Explicit and recalled in the "state-chart" for special operations required by the algorithm:

- two services in the global analysis for:

- the iterative correction over $\Gamma_{g}^{h}$, to implement what will be described in 3.3.2,

- the upscaling and replacement of the local solution in $\Omega_{g s}^{h}$, to implement what will be described in 3.3.3;

- two services in the local analysis for:

- the imposition of velocity constraint over $\Gamma_{\ell}^{h}$, to implement what will be described in 3.3.2,

- the imposition of external loads coming from the global analysis over $\Gamma_{\ell}^{h, \text { ext }}$, to implement what will be described in 3.3.4.

Mapping of exchanged quantities are automatically treated by the cosimulation mapper, with the exception of contact mapping, which required new implementations.

Finally, the iterative repetition of the sub-cycling step at the local level requires the implementation of the new capability to go back in time, saving nodal and state quantities at a given instant of the analysis and retrieving them for the subsequent iterations. As depicted in Figure 10, where $i$ denotes the current iteration, the local analysis needs to store quantities at time $t^{n}$ in order to restart the sub-cycling at iteration $i+1$.

\subsubsection{Iterative algorithm through the global-local interface}

The substitution algorithm steps from Bettinotti et al. (2014b), as used in the present implementation of the method, can be summarized as follows: 


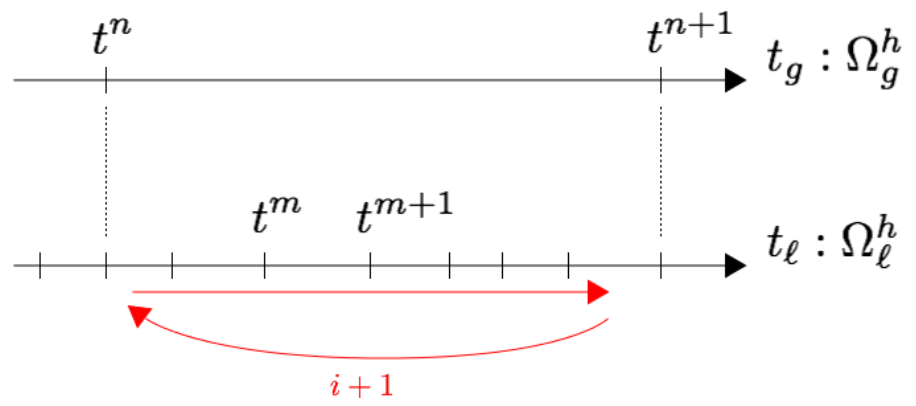

Figure 10: Iterations of the local sub-cycling require the capability of going back in time.

1. explicit computation in $\Omega_{g}^{h} \equiv \Omega_{g s}^{h} \cup \Omega_{g c}^{h}$ at a given time increment $\Delta t_{g}=t^{n+1}-t^{n}$

(a) half-time global nodal velocities ${ }^{n+1 / 2} \mathbf{V}_{g}$ and displacements ${ }^{n+1} \mathbf{U}_{g}$ are updated based on the central difference scheme;

(b) global momentum balance is enforced as

$$
\mathbf{M}_{g}\left({ }^{n+1} \mathbf{A}_{g}\right){ }^{0}={ }^{n+1} \mathbf{F}_{g}^{\mathrm{ext}}-{ }^{n+1} \mathbf{F}_{g}^{\mathrm{int}}
$$

where $\mathbf{M}_{g}$ is the lumped global mass matrix, $\left({ }^{n+1} \mathbf{A}_{g}\right)^{0}$ is the initial estimate of the global acceleration at the end of the global time increment, ${ }^{n+1} \mathbf{F}_{g}^{\text {ext }}$ are the global external nodal forces, which include also possible contact forces on $\Gamma_{g s}^{h, \text { ext }}$ and ${ }^{n+1} \mathbf{F}_{g}^{\text {int }}$ are internal equivalent nodal forces, computed from the displacements ${ }^{n+1} \mathbf{U}_{g}$;

2. global-to-local mapping through interpolation of external and contact forces from the loaded boundary $\Gamma_{g s}^{h \text {,ext }}$ of the substitution region of the global problem to the corresponding boundary $\Gamma_{\ell}^{h, \text { ext }}$ of the local problem;

3. iteration counter $i$ is initialized to 1 ;

4. global-to-local mapping of velocities and accelerations from $\Gamma_{g}^{h}$ to $\Gamma_{\ell}^{h}$. Let $s(t)=\left(t-t^{n}\right) / \Delta t_{c}, 0 \leq s \leq 1, t^{n} \leq t \leq t^{n+1}$, be an intrinsic local time. At the local time instants $s$, local nodal velocities $\mathbf{V}_{\ell \Gamma}(s)$ are defined in terms of global velocities and accelerations as (see Bettinotti et al. (2014b) for the derivation of this quadratic interpolation starting 
from a cubic Hermitian interpolation in terms of global nodal velocities and accelerations):

$$
\mathbf{V}_{\ell \Gamma}(s)=\Pi_{h}^{1}\left({ }^{n+1} \mathbf{V}_{g \Gamma}\right)^{i}+\Delta t s \Pi_{h}^{1}\left({ }^{n} \mathbf{A}_{g \Gamma}\right)^{i}+\Delta t \frac{s^{2}}{2} \Pi_{h}^{1}\left({ }^{n+1} \mathbf{A}_{g \Gamma}-{ }^{n} \mathbf{A}_{g \Gamma}\right)^{i}
$$

where $\Pi_{h}^{1}$ is a spatial linear mapping operator that interpolates values from the global mesh to the local one and where ${ }^{n+1} \mathbf{V}_{g \Gamma}$ is defined according to the central difference integration scheme as

$$
{ }^{n+1} \mathbf{V}_{g \Gamma}={ }^{n+1 / 2} \mathbf{V}_{g \Gamma}+{ }^{n+1} \mathbf{A}_{g \Gamma} \frac{\Delta t_{g}}{2},
$$

5. explicit computation in $\Omega_{\ell}^{h}$ (sub-cycling) with local time increments $\Delta t_{\ell}=t^{m+1}-t^{m}$ as in Figure 8, imposing the interpolated interface velocities (3) over $\Gamma_{\ell}^{h}$ as boundary conditions and contact and external forces on $\Gamma_{\ell}^{h, \text { ext }}$ as loads:

(a) local half-time velocities $\left({ }^{m+1 / 2} \mathbf{V}_{\ell}\right)^{i}$ and displacements $\left({ }^{m+1} \mathbf{U}_{\ell}\right)^{i}$ are computed following the central difference scheme;

(b) local momentum balance is enforced in the local region as

$$
\mathbf{M}_{\ell}\left({ }^{m+1} \mathbf{A}_{\ell}\right)^{i}=\left({ }^{m+1} \mathbf{F}_{\ell}^{\mathrm{ext}}\right)^{i}-\left({ }^{m+1} \mathbf{F}_{\ell}^{\mathrm{int}}\right)^{i}
$$

6. iteration counter is updated $i \leftarrow i+1$;

7. correction of global accelerations on $\Gamma_{g}^{h}$ by enforcing dynamic equilibrium of the interface between the local and complementary regions, under the action of inertia, external and internal equivalent nodal forces contributed by the local and complementary regions.

According to the multi-scale compatibility condition proved in Bettinotti et al. (2014b), one has that

$$
\left({ }^{n+1} \mathbf{A}_{\ell \Gamma}\right)^{i}=\Pi_{h}^{1}\left({ }^{n+1} \mathbf{A}_{g \Gamma}\right)^{i}
$$

Interface equilibrium requires therefore that (the subscript $g c \Gamma$ denotes quantities pertinent to nodes of the complementary region $\Omega_{g c}^{h}$ belonging to the interface $\left.\Gamma_{g}^{h}\right)$ :

$$
\tilde{\mathbf{M}}_{g \Gamma}\left({ }^{n+1} \mathbf{A}_{g \Gamma}\right)^{i}=\Pi_{h}^{1 T}{ }^{n+1} \mathbf{F}_{\ell \Gamma}^{\mathrm{ext}}+{ }^{n+1} \mathbf{F}_{g c \Gamma}^{\mathrm{ext}}-\Pi_{h}^{1 T}\left({ }^{n+1} \mathbf{F}_{\ell \Gamma}^{\mathrm{int}}\right)^{i}-{ }^{n+1} \mathbf{F}_{g c \Gamma}^{\mathrm{int}}
$$


where $\Pi_{h}^{1 T}$ is the linear spatial upscaling operator and $\tilde{\mathbf{M}}_{g \Gamma}$ is a narrowbanded, non-diagonal mass matrix defined as

$$
\tilde{\mathbf{M}}_{g \Gamma}=\Pi_{h}^{1 T} \mathbf{M}_{\ell \Gamma} \Pi_{h}^{1}+\mathbf{M}_{g c \Gamma} .
$$

8. go to step 4 if $i<3$ or go to step 1 otherwise; extensive numerical experiments have shown that this number of iterations returns an acceptable error in the equilibrium between global and local analyses.

Two co-simulation services have been written to implement this iterative

algorithm on the interfaces $\Gamma_{g}^{h}$ and $\Gamma_{\ell}^{h}$ : one for the global correction over $\Gamma_{g}^{h}$ (step 7 above), one for the boundary conditions imposition over $\Gamma_{\ell}^{h}$ (step 4 above).

The co-simulation quantities to be exchanged at each iteration through co-simulation are velocities and accelerations from global to local and interface forces and mass from local to global, such that:

$$
\begin{gathered}
\Gamma_{g}^{h} \rightarrow \Gamma_{\ell}^{h}:{ }^{n+1} \mathbf{V}_{g \Gamma},{ }^{n+1} \mathbf{A}_{g \Gamma} ; \\
\Gamma_{\ell}^{h} \rightarrow \Gamma_{g}^{h}: \mathbf{M}_{\ell \Gamma},{ }^{n+1} \mathbf{F}_{\ell \Gamma}^{\text {int }},{ }^{n+1} \mathbf{F}_{\ell \Gamma}^{\mathrm{ext}} .
\end{gathered}
$$

\subsubsection{Direct substitution}

In the interior of the global substitution region $\Omega_{g s i}^{h} \equiv \Omega_{g s}^{h} \backslash \Gamma_{g}^{h}$, velocities are simply mapped from the local nodes to the global ones. Local velocities are directly substituted in the global time integration and accelerations are calculated as:

$$
{ }^{n+1} \mathbf{A}_{g s i}=\left({ }^{n+1} \mathbf{V}_{\ell}-{ }^{n} \mathbf{V}_{g s i}\right) \frac{2}{\Delta t_{g}}-{ }^{n} \mathbf{A}_{g s i}
$$

An iterative correction of accelerations is not required in this region. The global-local equilibrium is iteratively searched only over the global-local interface $\Gamma_{g}^{h}$. In the global substitution region, the solution of interest is only the local one.

A co-simulation service has been implemented to perform the direct substitution in the global substitution region $\Omega_{g s}^{h}$. 
The quantity to be exchanged through co-simulation from local to global at the end of the iterations is the velocity:

$$
\Omega_{\ell}^{h} \rightarrow \Omega_{g s}^{h}:{ }^{n+1} \mathbf{V}_{\ell}
$$

\subsubsection{Contact global-to-local mapping}

A new mapper for the transmission of global contact forces to the local analysis has been integrated into the co-simulation services. Contact is here detected only in the global analysis, while equivalent contact forces are passed to the local analysis. This approach is considered less intrusive, because in this way contact is treated at the global scale only, using the standard code capabilities, while the local model will focus only on the composite panel, and more performing, because contact will not be dealt with at the finer scale. The proposed method alleviates a drawback of domain decomposition approach when different time increments are used in different domain. Indeed, for such method, a constraint expanding over those different domains would require the use of the finer associated time increment.

Figure 11 shows the contact mapping scheme:

1. contact is detected during the global analysis with the coarse mesh and global contact forces are estimated via the penalty method;

2. the global mesh is subdivided into a sub-mesh with smaller elements;

3. global contact forces are transformed into uniform pressures over the new elements of the global sub-mesh,

4. the pressure load is mapped onto the local mesh, integrated and then applied in the form of equivalent local nodal forces.

Such scheme does not conserve the resultant of the contact forces and the unbalance is finally equally redistributed over the local nodes in order to preserve the balance between global contact forces and the local mapped ones. Despite this complication, the mapping simplifies the local model and it is not computationally expensive, since it exploits the frequent data exchange between global and local analyses.

Furthermore, the global and local surfaces will be misaligned at the end of the analysis, because velocity compatibility between the two scales is not enforced on the loaded boundary $\Gamma_{g s}^{h, e x t}$. 

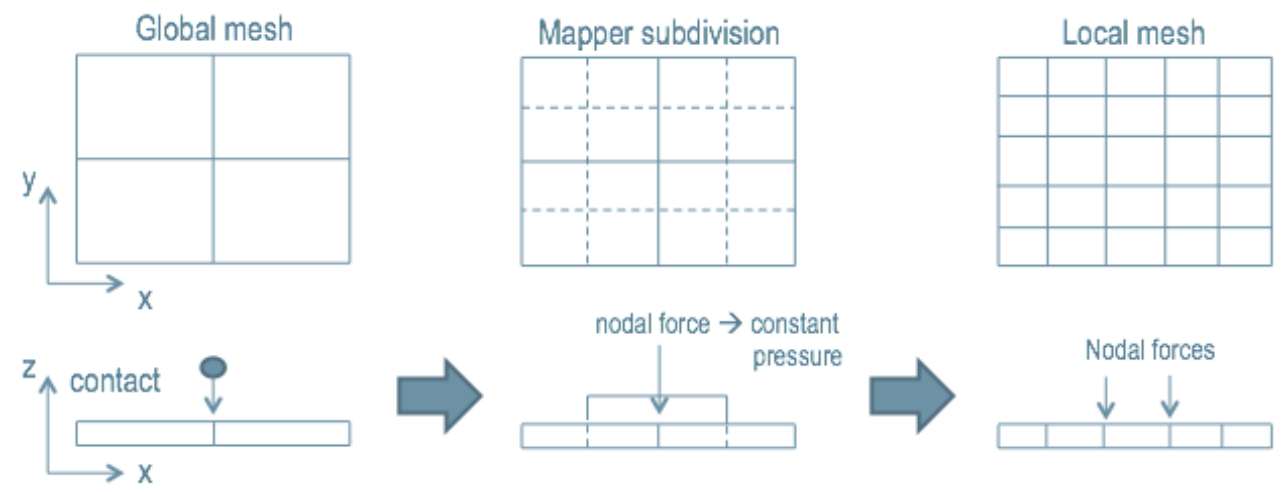

Figure 11: Contact mapping from global to local analysis.

The contact mapping described in this Section 3.3.4 would be very computationally intensive for the sub-modeling technique described in section 3.2 available in Abaqus/Explicit as a standard feature, because of the large amount of contact data to be stored during the global analysis.

A co-simulation service has been implemented to perform the mapping of the contact forces to the local surface $\Gamma_{\ell}^{h, \text { ext }}$.

The quantities to be exchanged at each iteration through co-simulation are contact and external forces from global to local, such that:

$$
\Gamma_{g}^{h, \text { ext }} \rightarrow \Gamma_{\ell}^{h, \text { ext }}:{ }^{n+1} \mathbf{F}_{g}^{\text {ext }}
$$

\subsubsection{Global-to-local mapping in time}

Since a smaller time increment is used for the local analysis, a linear interpolation in time is applied to the nodal contact and external forces $\mathbf{F}_{g s}^{\text {ext }}$ across the two time scales:

$$
{ }^{m+1} \mathbf{F}_{\ell}^{\mathrm{ext}}=\Pi_{t}^{1}\left({ }^{n} \mathbf{F}_{g s}^{\mathrm{ext}},{ }^{n+1} \mathbf{F}_{g s}^{\mathrm{ext}}\right),
$$

where the downscaling operator $\Pi_{t}^{1}$ indicates linear interpolation in time, so that Equation (10) becomes

$$
{ }^{m+1} \mathbf{F}_{\ell}^{\mathrm{ext}}=\frac{t^{n+1}-t^{m+1}}{\Delta t_{g}}{ }^{n} \mathbf{F}_{g s}^{\mathrm{ext}}+\frac{t^{m+1}-t^{n}}{\Delta t_{g}}{ }^{n+1} \mathbf{F}_{g s}^{\mathrm{ext}},
$$

where time definitions are taken from Figure 8. 


\section{Results}

The test case is analyzed here for the verification of the substitution method. Four methods are compared in this study:

- uniform mesh with pseudo-meso-scale model applied everywhere;

- non-uniform mesh with non-overlapped multi-scale model:

- pseudo-meso-scale model applied to the impacted zone;

- macro-scale model applied to the rest of the panel;

- tie constraint to merge the two regions in the same analysis;

- multi-scale sub-modeling technique:

- global analysis with macro-scale model applied to the overall panel;

- local analysis with pseudo-meso-scale model applied to the impacted zone, including the impactor;

- multi-scale substitution via co-simulation technique:

- global analysis with macro-scale model applied to the overall panel;

- local analysis with pseudo-meso-scale model applied to the impacted zone, without impactor and contact model.

Some details of the analyses in terms of number of elements $n$ and number of time increments $N$ are given in Table 1 . The number of elements

\begin{tabular}{lccc}
\hline Method & Model & $n$ elements & $N$ time increments \\
\hline uniform analysis & pseudo-meso & 77246 & 1583 \\
tie constraint & macro/pseudo-meso & 20734 & 1583 \\
sub-modeling & macro/pseudo-meso & $5246+18936$ & $219+1583$ \\
substitution & macro/pseudo-meso & $5246+18936$ & $223+1660$ \\
\hline
\end{tabular}

Table 1: Details about the methods and models used for the verification of the substitution method applied to the test case.

considerably decreases with the use of multi-scale coupling techniques when compared to the uniform finite element analysis. Note that the elements in 
the macro-scale model are considered as linear elastic with the pre-integrated section and the related computational cost is considerably cheaper than the pseudo-meso-scale model, in which non-linearities are modeled. So, for submodeling and substitution, the important indicator for performance is the number of elements in the pseudo-meso-scale model.

\subsection{Concentrated load at the middle of the panel}

At first, the test case is analyzed without impact, with a sharp concentrated load at the middle of the plate with the history amplitude reported in Table 2.

\begin{tabular}{cccccc}
\hline time $[m s]$ & 0 & 0.01 & 0.05 & 0.06 & 0.2 \\
load $[k N]$ & 0 & -20 & -20 & 0 & 0 \\
\hline
\end{tabular}

Table 2: History amplitude of the concentrated load.

Figure 12 shows results for the four different methods in terms of displacements at the middle of the analysis (Figure 12a) and at the end of the analysis (Figure 12b). For sub-modeling and substitution, only the results of the local analysis are presented. In the middle of the analysis, uniform mesh analysis, tie constraint and substitution are aligned on the same results, whereas sub-modeling has reasonable results around the loaded location but presents some wave-reflection noise along top and left borders of the local domain. Such noise propagates and increases towards the end of the analysis, for sub-modeling more than for tie constraint and substitution, as shown in Figure 12b. For the tie constraint, a wave-reflection along the right edge of the pseudo-meso-scale model is remarked with higher values of displacement. For the substitution, some wave-reflection of the bottom-right of the local domain is remarked and the displacement solution around the loaded zone is not regularly round as in the reference uniform mesh results. Note that for the tie constraint the smallest time increment is used everywhere, while for the substitution method the smallest time increment is employed only in the local domain; which explains why the results are slightly different.

The better behavior of substitution method in the presence of wavereflections due to different spatial and temporal discretizations in global and local analyses is explained by the velocity histories in Figure 13. The difference rests in the mapping interpolation process in time from the global 


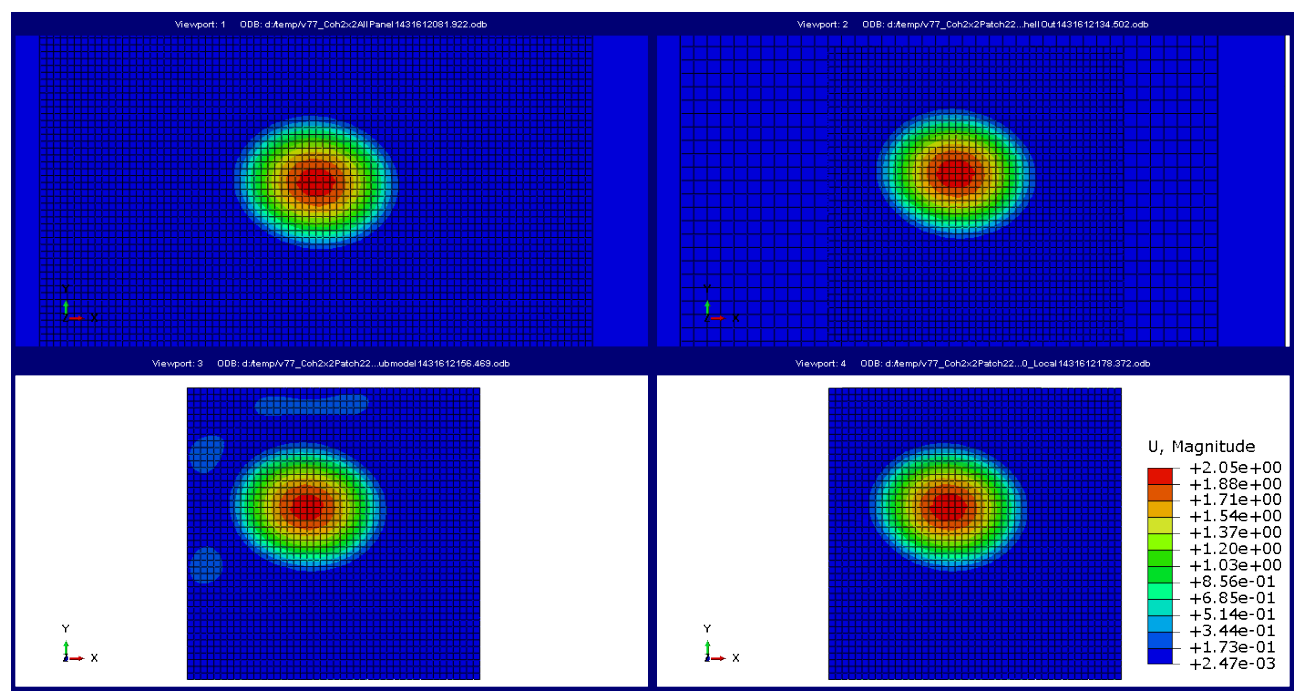

(a) $t=0.1 \mathrm{~ms}$

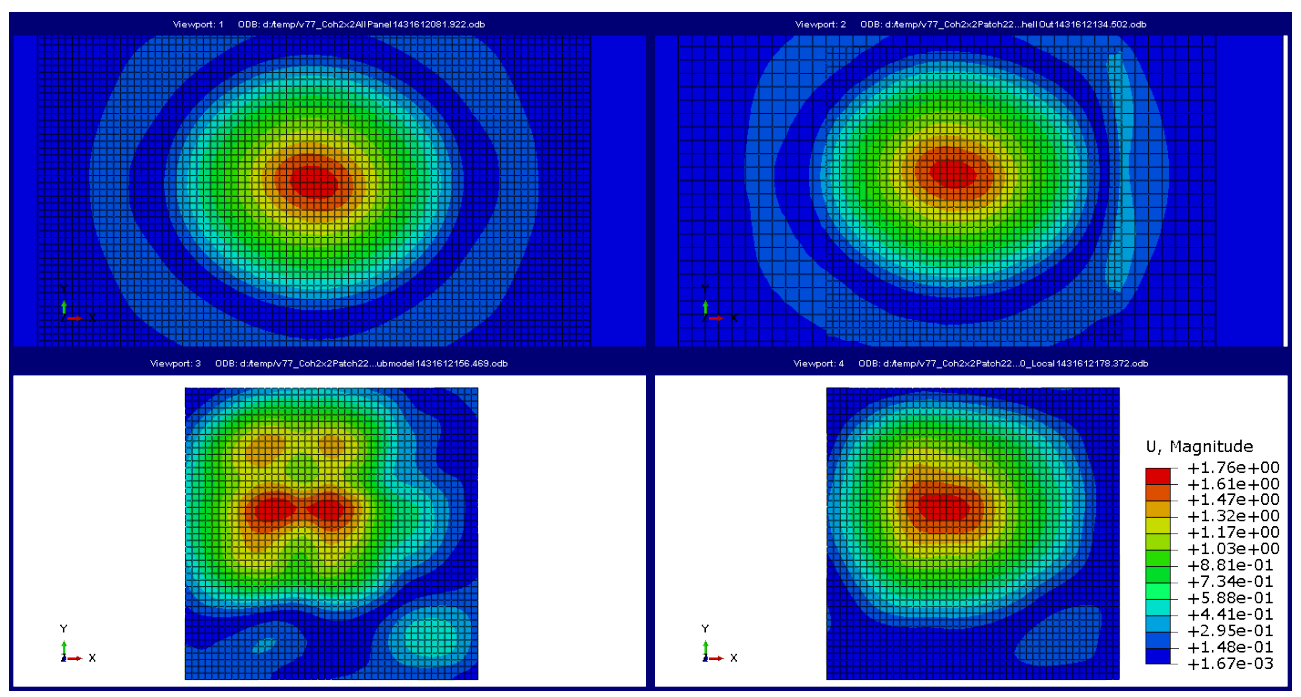

(b) $t=0.2 \mathrm{~ms}$

Figure 12: Concentrated load test case: transverse displacement fields. From left to right, from top to bottom: uniform mesh analysis, tie constraint, sub-modeling, substitution. 
analysis to the local one. Velocities over time are traced in Figure 13a for sub-modeling and in Figure 13b for substitution at a coincident location over the global-local interface. In the first case, with sub-modeling, either a linear or a quadratic interpolation is used by Abaqus, depending on criteria internally implemented. The choice of the interpolation order may be different for different time increments. In terms of velocities, the result will be either piece-wise linear or constant in time, over many time increments. In the second case, with substitution method, a quadratic interpolation of velocities is performed at each global time increment, using also nodal values of global accelerations. As a result, the local velocity shape (green line) is smooth in time. In Figure 13 all the iterations of the algorithm are considered and only the first local iteration is out of the global corrected solution (the orange line then returns at the beginning of the subcycling to reiterate the local solution).

Figure 14 shows results of the same analysis in terms of delamination. The shape of the delaminated zone is caught well by all the methods, but sub-modeling and tie constraint detect delamination over the borders of the pseudo-meso-scale model due to wave-reflection noise. Substitution exhibits less noised results.

\subsection{Impact test case}

Figure 15 shows results related to the impact test case, once again for the four different methods, in terms of displacements at the middle of the analysis (Figure 15a) and at the end of the analysis (Figure 15b). Results are for this case smoother. All the four analyses predict the plate displaced configuration with acceptable accuracy.

The most important differences are related to the prediction of delamination, shown in Figure 16. The shape of the delaminated zone is caught well by all the methods. Once again, sub-modeling, tie constraint and substitution detect delamination over the borders of the pseudo-meso-scale model due to wave-reflection noise. At time $0.05 \mathrm{~ms}$, tie constraint and sub-modeling present some delamination noise over the top left corner of the delaminated area, whereas substitution presents results more in line with the uniform mesh analysis. Such result is appreciable, in view of the approximated contact mapping in the substitution method. Such noise is then more evident at time $0.2 \mathrm{~ms}$, especially in the sub-modeling results. Due to wave-reflection, the tie constraint produces large delamination errors at the right edge of the 


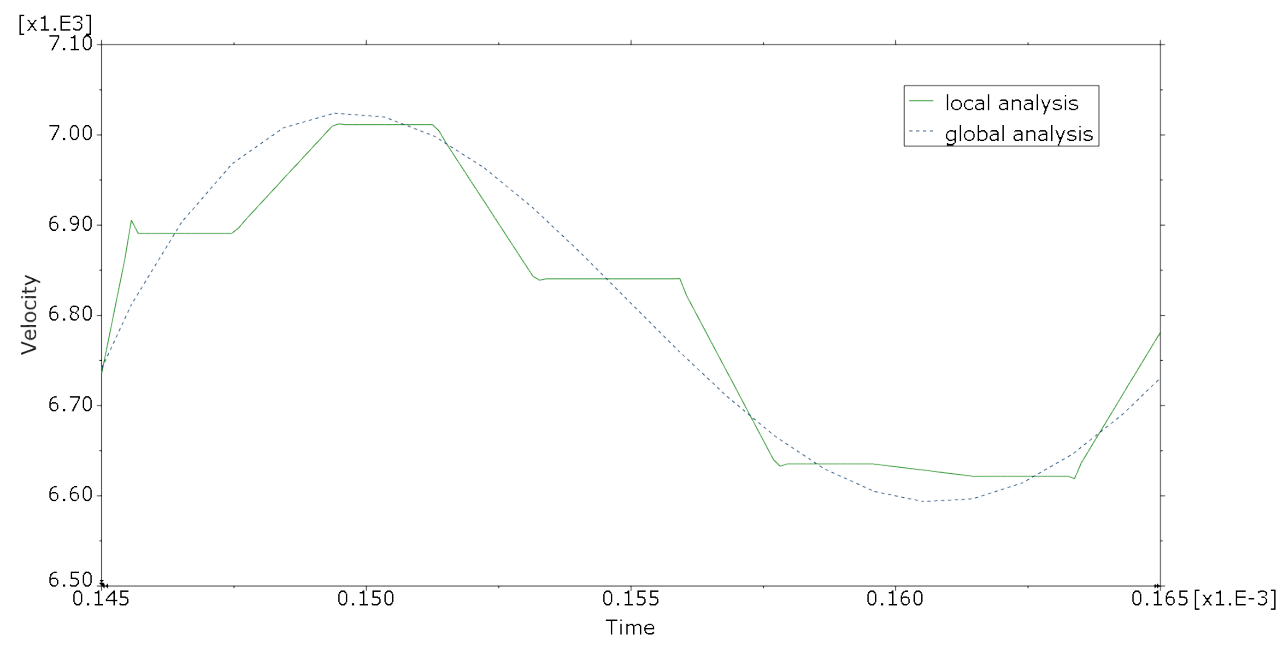

(a) sub-modeling

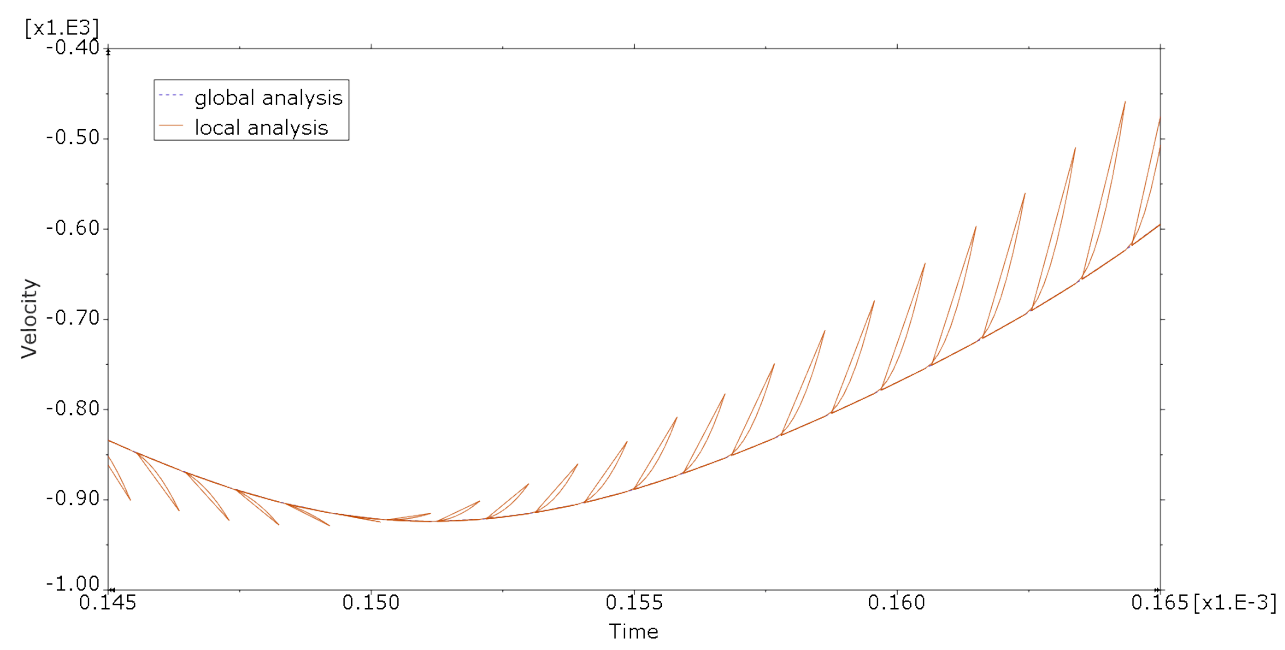

(b) substitution

Figure 13: Velocity histories at a location over the interface. 


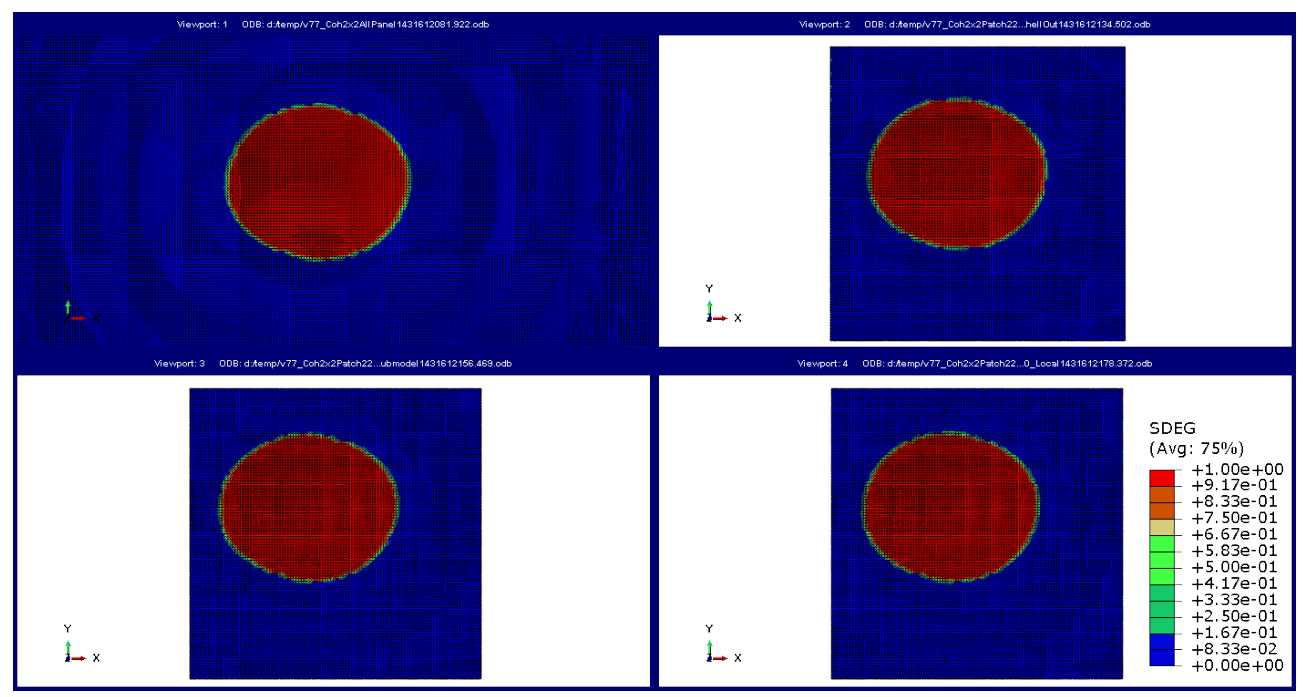

(a) $t=0.1 \mathrm{~ms}$

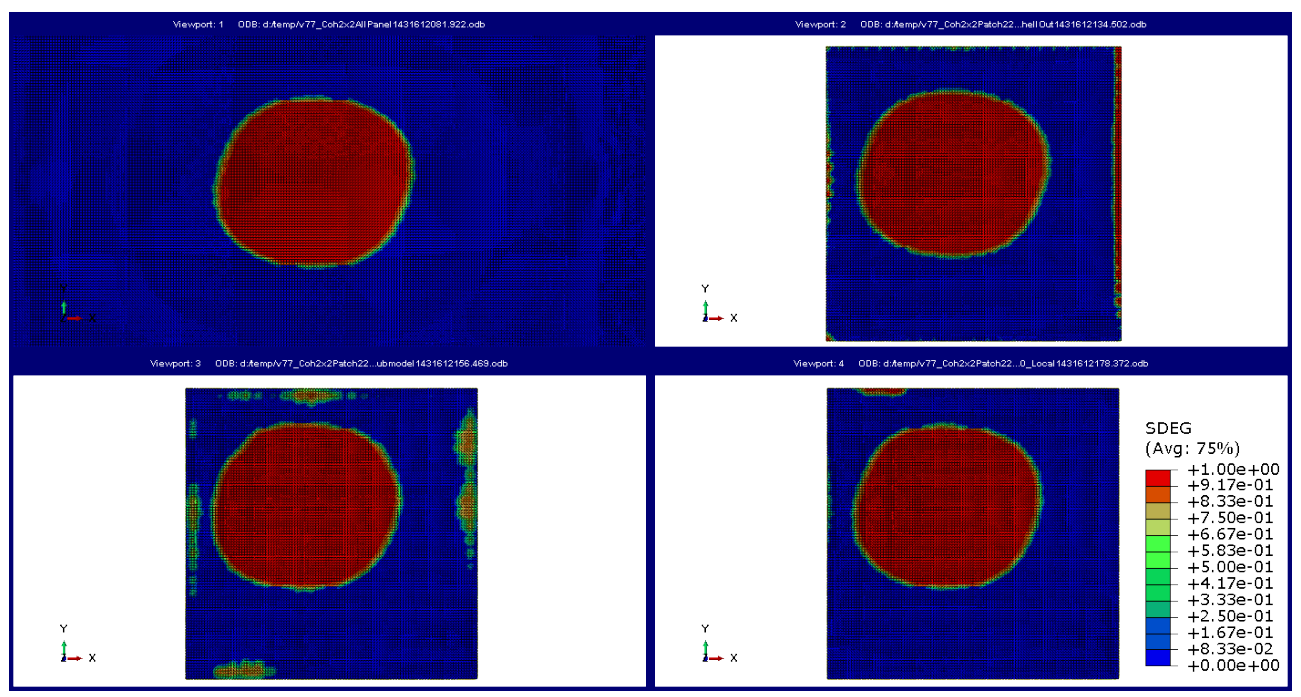

(b) $t=0.2 \mathrm{~ms}$

Figure 14: Concentrated load test case: delamination propagation. From left to right, from top to bottom: uniform mesh analysis, tie constraint, sub-modeling, substitution. 


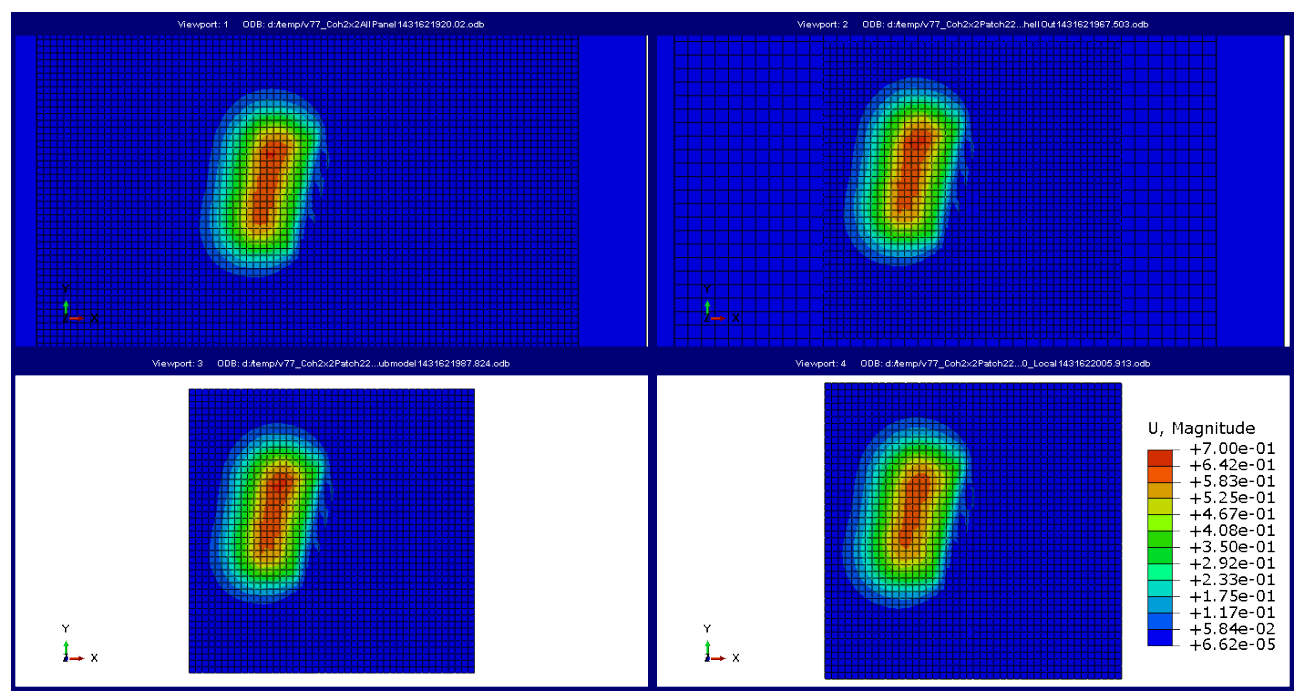

(a) $t=0.05 \mathrm{~ms}$

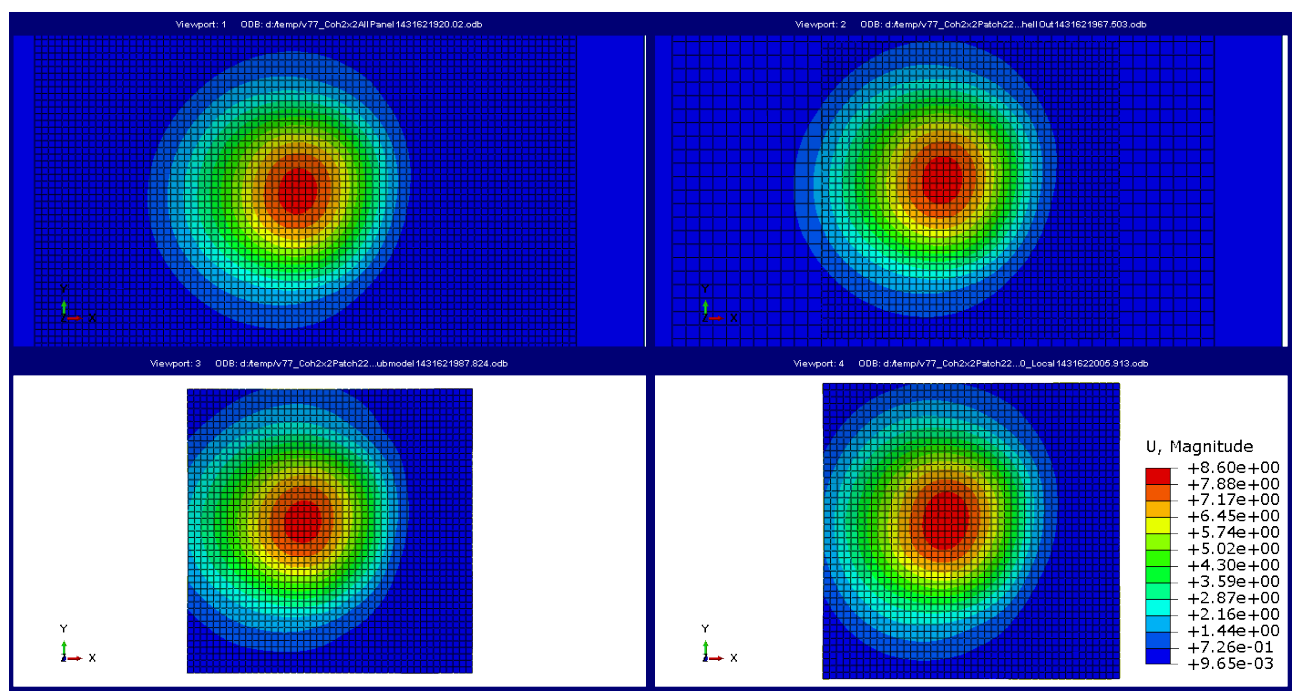

(b) $t=0.2 \mathrm{~ms}$

Figure 15: Impact test case: displacements field. From left to right, from top to bottom: uniform mesh analysis, tie constraint, sub-modeling, substitution. 


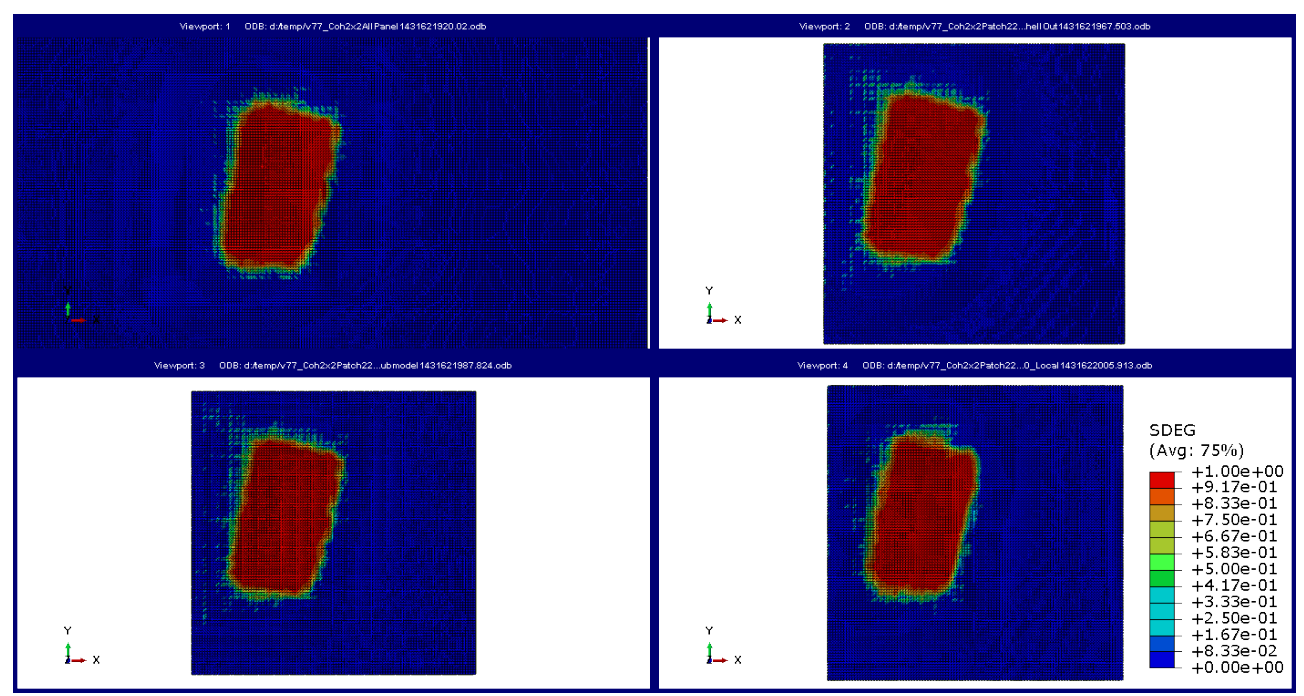

(a) $t=0.05 \mathrm{~ms}$

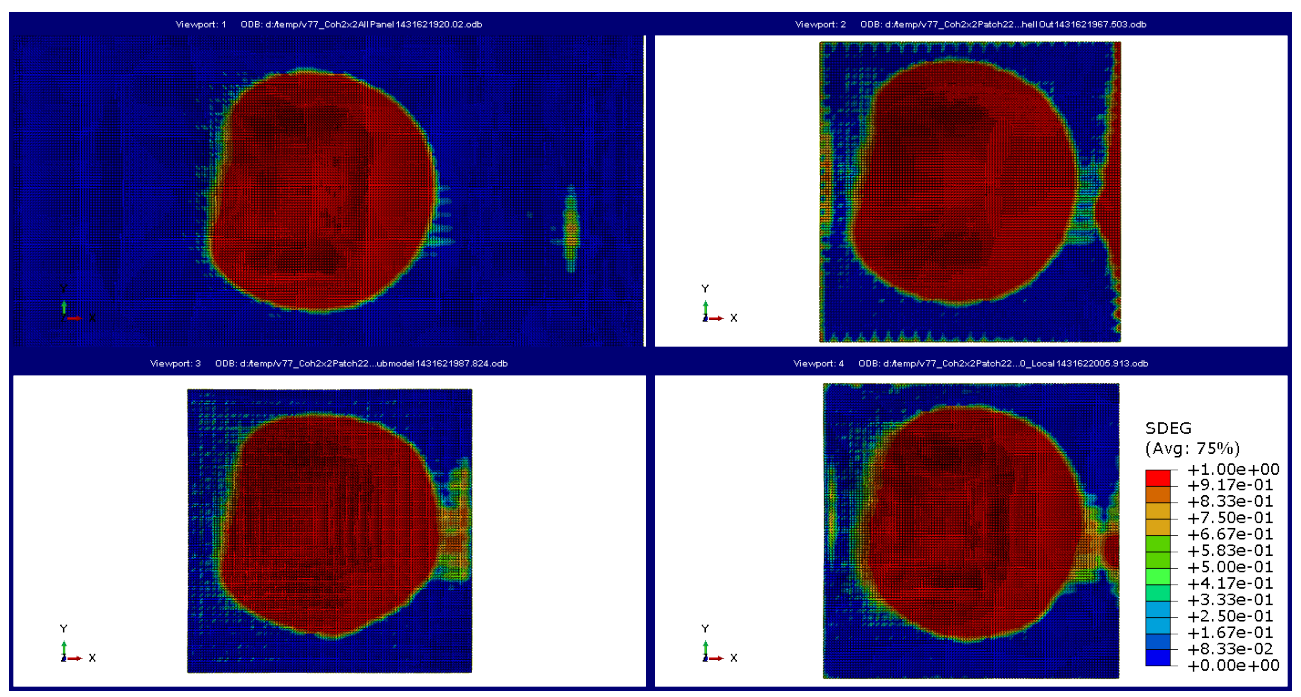

(b) $t=0.2 \mathrm{~ms}$

Figure 16: Impact test case: delamination propagation. From left to right, from top to bottom: uniform mesh analysis, tie constraint, sub-modeling, substitution. 
pseudo-meso-scale model, whereas sub-modeling and substitution manage to contain it.

\section{Conclusions and future prospects}

The paper concerns new developments of the substitution method introduced in Bettinotti et al. (2014a) and reformulated in a faster algorithm in Bettinotti et al. (2014b). This approach was previously implemented in a Matlab code and validated on simple examples in the context of linear elasticity. The paper describes new code implementations that have been required to integrate the algorithm into the Abaqus/Explicit application and into its co-simulation services. This has allowed to treat an example of industrial interest, the one of an impact test on a stratified composite panel leading to delamination. The effectiveness of the method has been verified by comparing the results with other approaches already available in Abaqus/Explicit: the one based on the use of a tie constraint between meshes of different fineness and the sub-modeling approach. The results show a good accuracy of the substitution method. One key aspect of the method, if compared to one based on domain decomposition, is that the global model covers the whole structure. This feature has been used to implement the method in the most simple manner by introducing the contact constraint at the global level only.

In the case of an analysis duration longer than the one considered in this paper, the next challenge to improve the method concerns the possibility to consider a local patch evolving according to transient phenomena such as, e.g., the propagation of delamination. To achieve this, multi-scale coupling methods, like the domain decomposition method, would have to be combined with re-meshing strategies, which however are considered to be intrusive in terms of their implementation within a legacy finite element software. In contrast, the proposed method would allow one to keep unmodified the coarse model and to adapt the fine local model on the fly, depending on an activation criterion to be defined.

Another possible future development concerns the improvement of the treatment of the global-local interface, in order to alleviate spurious wavereflections, as proposed for instance in To and Li (2005), or by using artificial dissipative techniques alternative to bulk viscosity, such as those introduced for fluid dynamics in VonNeumann and Richtmyer (1950) and Landshoff (1955). Moreover, it would be very useful to introduce the possibility to make use of geometrically non-conforming global-local meshes, considering global- 
local interfaces that are not aligned with the global elements boundaries. For this purpose, special interpolation functions could be used, as for instance those proposed in Biabanaki et al. (2014) that considers polygonal elements for large deformations contact problems.

\section{References}

Abaqus, 2014. Abaqus v6.14 Analysis User's Guide. Dassault Systèmes SIMULIA.

Allix, O., 2001. A composite damage meso-model for impact problems. Composites Science and Technology 61, 2193-2205.

Belytschko, T., Yen, H.-J., Mullen, R., 1979. Mixed methods for time integration. Computer Methods in Applied Mechanics and Engineering 17-18, 259-275.

Bettinotti, O., Allix, O., Malherbe, B., 2014a. A coupling strategy for adaptive local refinement in space and time with a fixed global model in explicit dynamics. Computational Mechanics 53, 561-574.

Bettinotti, O., Allix, O., Perego, U., Oancea, V., Malherbe, B., 2014b. A fast weakly intrusive multiscale method in explicit dynamics. International Journal for Numerical Methods in Engineering 100 (8), 577-595.

Biabanaki, S., Khoei, A., Wriggers, P., 2014. Polygonal finite element methods for contact-impact problems on non-conformal meshes. Computer Methods in Applied Mechanics and Engineering 269, 198 - 221.

Boucard, P. A., Odievre, D., Gatuingt, F., 2002. A 3d shock computational strategy for real assembly and shock attenuator. Advances in Engineering Software 33, 517-526.

Boucard, P. A., Odievre, D., Gatuingt, F., 2011. A parallel and multiscale strategy for the parametric study of transient dynamic problems with friction. International Journal for Numerical Methods in Engineering 88, 657672 .

Chantrait, T., Rannou, J., Gravouil, A., 2014. Low intrusive coupling of implicit and explicit time integration schemes for structural dynamics: Application to low energy impacts on composite structures. Finite Elements in Analysis and Design 86, 23-33. 
Combescure, A., Gravouil, A., 2002. A numerical scheme to couple subdomains with different time-steps for predominantely transient linear analysis. Computer Methods in Applied Mechanics and Engineering 191 (11-12), $1129-1157$.

Daniel, W. J. T., 2003. A partial velocity approach to subcycling structural dynamics. Computer Methods in Applied Mechanics and Engineering $192(3), 375-394$.

Esgandari, M., Olatunbosun, O., 2015. Implicit-explicit co-simulation of brake noise. Finite Elements in Analysis and Design 99, 16-23.

Farhat, C., Crivelli, L., Roux, F.-X., 1994. A transient FETI methodology for large-scale parallel implicit computations in structural mechanics. International Journal for Numerical Methods in Engineering 37 (11), 1945-1975.

Farhat, C., Roux, F.-X., 1991. A method of finite element tearing and interconnecting and its parallel solution algorithm. International Journal for Numerical Methods in Engineering 32, 1205-1227.

Gendre, L., Allix, O., Gosselet, P., 2011. A two-scale approximation of the Schur complement and its use for non-intrusive coupling. International Journal for Numerical Methods in Engineering 87, 889-905.

Gendre, L., Allix, O., Gosselet, P., Comte, F., 2009. Non-intrusive and exact global/local techniques for structural problems with local plasticity. Computational Mechanics 44 (2), 233-245.

Gigliotti, L., Pinho, S., 2015. Multiple length/time-scale simulation of localized damage in composite structures using a mesh superposition technique. Composite Structures 121, 395-405.

Gravouil, A., Combescure, A., 2001. Multi-time-step explicit-implicit method for non-linear structural dynamics. International Journal for Numerical Methods in Engineering 50, 199-225.

Guguin, G., Allix, O., Gosselet, P., Guinard, S., 2014. Nonintrusive coupling of $3 \mathrm{D}$ and $2 \mathrm{D}$ laminated composite models based on finite element $3 \mathrm{~d}$ recovery. International Journal for Numerical Methods in Engineering 98 (5), 324-343. 
Gupta, P., Pereira, J. P., Kim, D.-J., Duarte, C. A., Eason, T., 2012. Analysis of three-dimensional fracture mechanics problems: A non-intrusive approach using a generalized finite element method. Engineering Fracture Mechanics 90, 41-64.

Hashin, Z., Rotem, A., 1973. A fatigue failure criterion for fiber-reinforced materials. Journal of Composite Materials 7, 448-464.

Kerfriden, P., Passieux, J. C., Bordas, S. P. A., 2012. Local/global model order reduction strategy for the simulation of quasi-brittle fracture. International Journal for Numerical Methods in Engineering 89 (2), 154-179.

Ladevèze, P., Loiseau, O., Dureisseix, D., 2001. A micro-macro and parallel computational strategy for highly heterogeneous structures. International Journal for Numerical Methods in Engineering 52 (1-2), 121-138.

Ladevèze, P., Nouy, A., 2003. On a multiscale computational strategy with time and space homogenization for structural mechanics. Computer Methods in Applied Mechanics and Engineering 192, 3061-3087.

Landshoff, R., 1955. A numerical method for treating fluid flow in the presence of shocks. Technical Report LA-1930, Los Alamos National Laboratory, $1-40$.

Mandel, J., 1993. Balancing domain decomposition. International Journal for Numerical Methods in Biomedical Engineering 9 (3), 233-241.

Nour-Omid, B., Wriggers, P., 1986. A two-level iteration method for solution of contact problems. Computer Methods in Applied Mechanics and Engineering 54, 131-144.

Passieux, J.-C., Réthoré, J., Gravouil, A., Baietto, M.-C., 2013. Local/global non-intrusive crack propagation simulation using a multigrid X-FEM solver. Computational Mechanics 52 (6), 1381-1393.

Plews, J., Duarte, C. A., Eason, T., 2012. An improved nonintrusive globallocal approach for sharp thermal gradients in a standard FEA platform. International Journal for Numerical Methods in Engineering 91 (4), 361397. 
Sicklinger, S., Belsky, V., Engelmann, B., Elmqvist, H., Olsson, H., Wüchner, R., Bletzinger, K.-U., 2014. Interface jacobian-based co-simulation. International Journal for Numerical Methods in Engineering 98, 418-444.

To, A. C., Li, S. F., 2005. Perfectly matched multiscale simulations. Physical Review B 72 (035414), 1-8.

VonNeumann, J., Richtmyer, R. D., 1950. A method for the numerical calculation of hydrodynamic shocks. Journal of Applied Physics 21, 232-237.

Whitcomb, J. D., 1991. Iterative global/local finite element analysis. Computers and Structures 40 (4), 1027-1031. 